\title{
Corela
}

Cognition, représentation, langage

HS-1 | 2005

Colloque AFLS

\section{Le présent de reportage dans la presse quotidienne}

\section{Bénédicte Facques}

\section{(2) OpenEdition}

Journals

Édition électronique

URL : http://journals.openedition.org/corela/1151

DOI : 10.4000/corela. 1151

ISSN : 1638-573X

Éditeur

Cercle linguistique du Centre et de l'Ouest - CerLICO

\section{Référence électronique}

Bénédicte Facques, «Le présent de reportage dans la presse quotidienne », Corela [En ligne], HS-1 | 2005, mis en ligne le 16 février 2005, consulté le 17 juin 2020. URL : http://journals.openedition.org/ corela/1151; DOI : https://doi.org/10.4000/corela.1151

Ce document a été généré automatiquement le 17 juin 2020.

\section{(c) (i) (2) (2)}

Corela - cognition, représentation, langage est mis à disposition selon les termes de la licence Creative Commons Attribution - Pas d'Utilisation Commerciale - Partage dans les Mêmes Conditions 4.0 International. 


\title{
Le présent de reportage dans la presse quotidienne
}

\author{
Bénédicte Facques
}

\section{Introduction}

1 En étudiant les textes de reportages de la presse écrite, j'ai constaté l'emploi massif du présent dans ce genre discursif où il représente plus de la moitié des formes employées. Plutôt que de mettre cette domination sur le compte de la polyvalence temporelle du présent qui peut exprimer toutes les époques, j'ai formé l'hypothèse d'un emploi spécifique du présent dans les reportages, emploi que j'appelerai " présent de reportage » (PR de R). Dans la tradition linguistique (Tasmowski-De-Ryck 1985, Mellet 1998), ce terme de "présent de reportage » est réservé à usage oral de cette forme verbale lorsque le présent est utilisé pour les commentaires en direct d'événements sportifs par exemple. A l'écrit, l'information est nécessairement différée, ce qui implique un changement de système temporel du présent vers le passé. On constate ainsi que le passé simple ou l'imparfait narratif sont fréquemment utilisés dans les reportages sportifs écrits. Mais pour la majorité des auteurs, le seul tiroir qui transpose vraiment les qualités du PR de R dans les récits écrits est le présent historique $(\mathrm{PH})$ qui, selon la formule consacrée, permet de «rapporter les événements comme s'ils étaitent présents ». Le présent historique se rencontre effectivement dans les reportages de presse écrite mais on trouve également une autre valeur de présent pour laquelle je maintiens le terme de PR de R car ses caractéristiques sont très proches de celles que l'on peut identifier pour les reportages oraux sur le plan du repérage temporel des procès, de la perspective temporelle du récit et de l'accès cognitif à l'information. Ce sont ces trois point que je me propose de développer pour mettre en évidence le PR de R et le distinguer du PH. Comme son nom l'indique, le PR de R se rencontre dans les textes de reportage (et plus largement dans tout type de récit d'événements attestés), c'est donc ce type de textes que j'ai sélectionné comme base de mon corpus dans quatre quotidiens de la presse nationale (Le Monde, Libération, l'Humanité et Le Figaro) pendant les mois de septembre 1997 et mai 1998. Le 
critère définitoire du reportage étant la présence du journaliste sur les lieux mêmes de l'événement (Voirol 1995), j'ai retenu tous les textes comportant une formule de suscription : de + nom du journaliste ou qualité + nom de ville ou nom de pays, par exemple « de notre envoyé spécial à Rome ».

\section{Le repérage temporel des procès}

Le repérage temporel renvoie à la manière dont les procès sont repérés par rapport au repère de l'actualité. Les événements peuvent être antérieurs, postérieurs ou coïncider avec ce repère de l'actualité. La spécificité du reportage de presse écrite est qu'il dispose d'un double repère de l'actualité. Le premier repère de l'actualité est matérialisé par la date de parution du journal : c'est le repère-origine conventionnel de la plupart des textes de presse écrite où le scripteur pose son énonciation comme contemporaine du moment de la réception. Le second repère de l'actualité correspond au repère de l'événement lorsque le scripteur pose son énonciation comme contemporaine du moment de l'événement. Ce déplacement du repère de l'actualité, du moment de la réception au moment de l'événement, est possible et vraisemblable dans le reportage parce que le journaliste a été le témoin des événements qu'il décrit et qu'il a partagé les mêmes coordonnées énonciatives que les locuteurs qu'il met en scène. Le second repère de l'actualité qui correspond au repère de l'événement n'est plus explicité à l'heure actuelle et il doit être reconstitué à partir des indications temporelles intra-textuelles mais il était clairement indiqué dans les reportages du passé. En consultant des reportages à l'étranger du Figaro, du Monde et de l'Humanité1, des années 1950 et 1960, j'ai en effet constaté que la formule de suscription mentionnait non seulement le lieu de production du reportage comme il est toujours d'usage à l'heure actuelle, mais également sa date de production qui ne correspondait pas à la date de parution du journal. La conséquence de ce double repérage est que, lorsque les événements sont racontés au présent, ces PR indiquent la coïncidence des procès avec la date de la formule de suscription et non avec la date de parution du journal :

(1) Taipeh, 6 février.- Un communiqué de l'armée de l'air nationaliste chinoise annonce que des B-24 et des B-25 ont effectué aujourd'hui plusieurs raids sur Changhaï, bombardant notamment le quartier industriel et l'usine électronique de Chapeï, les quais et l'aérodrome.

Le Figaro, 7 février 1950, «Violent bombardement de Changhaï par l'aviation nationaliste "

(2) Londres, 4 mars.- Même si les détails pittoresques [entourant la visite du président Auriol] occupent la plus grande place, certains journaux n'oublient pas la signification profonde de cette visite. Aujourd'hui même le News Chronicle consacre son éditorial à « la profonde et forte amitié franco-britannique ».

Le Monde, 5-6 mars 1950, «Londres se prépare à célébrer la visite du président Auriol»

Ainsi les déictiques « aujourd'hui » et " aujourd'hui même » dans les séquences (1) et (2) repèrent les procès en relation avec la date de la formule de suscription et non avec le repère origine conventionnel (date de publication du journal). Ce qui importe dans la presse écrite n'est donc pas tant le moment réel de l'énonciation que le moment où le locuteur pose son énonciation. Or, fait unique dans le discours journalistique, le reporter dispose de deux repères possibles pour poser son énonciation : la date de parution du journal (repère-origine 1) ou le moment de l'événement (repère-origine 2). La première différence entre le $\mathrm{PH}$ et le $\mathrm{PR}$ de $\mathrm{R}$ tient au fait que le premier repère les procès par 
rapport au repère-origine 1 et que cette relation entre le repère et le repéré est de type aoristique, tandis que le second repère les procès par rapport à un repère-origine fictif (repère-origine 2) et que la relation entre repère et repéré est une relation composite.

Le concept d'aoristique qui a été redéfini dans le domaine français par Culioli (1978:190) renvoie à l'opération de rupture (notée 㑌) entre le temps de l'événement ( $\mathrm{Te}$ ) et le temps $\mathrm{du}$ repère-origine (To) tel que $\mathrm{Te}$ 醫 $\mathrm{To}$. Dans le domaine temporel, l'aoristique est une catégorie dont les marqueurs peuvent être le passé simple, l'imparfait, le passé composé, le futur et le présent dans le cas du présent dit historique. Le récit au présent historique, construit par décrochage (ou rupture) par rapport à la situation d'énonciation origine (Sito) constitue en effet un exemple du mode de repérage aoristique. Mais à la différence des récits au PS ou au PC, dans lesquels ce sont les formes verbales qui marquent l'opération de rupture (qu'elle soit d'ordre temporel ou fictif), le présent en tant que tel n'indique pas ce décrochage qui est signifié par des indications temporelles non verbales avec lesquelles le PH entre en corrélation :

(3) C'est le 24 novembre 1965 que Joseph-Désiré Mobutu, alors âgé de trente-cinq ans, prend le pouvoir en renversant le président Joseph Kasavubu. L'ancien sergent de la « Force publique » du Congo sous colonisation belge, devenu journaliste, secrétaire particulier de Patrice Lumumba puis chef d'état-major des armées, tisse peu à peu sa toile sur l'ensemble du pays. Celui qui prétend incarner la nation élimine la vieille garde politique, interdit les partis, suspend le droit de grève, et s'applique à instituer l'unité du pays à son profit personnel. (...) Afin de bétonner encore un peu plus son pouvoir, il crée en 1967 le Mouvement populaire de la révolution (MPR, parti unique), et proclame l'« authenticité » dogme officiel du pays.

L'Humanité, 9/09/97, « La mort du maréchal-gangster Mobutu »

(4) Formé par Kang Sheng, l'ancien chef de l'espionnage du régime chinois, Qiao Shi a fait surface à Téhéran en 1978, lors d'une rencontre avec le chef de la police secrète iranienne. En 1982, il entre au Comité central et en 1987 dans la cellule de contrôle du régime, le Comité permanent du Bureau politique, qui ne compte alors que cinq membres. Au moment des événements de Tien Anmen, au printemps 1989, les hésitations de Qiao Shi face à la répression lui valent de se faire souffler la place de chef du parti, qu'il convoitait, par Jiang Zenim.

Libération, 19/09/97, « Le président chinois évince son grand rival »

5 Dans ces deux séquences, les indications temporelles fonctionnent comme des repères absolus et localisent les procès au $\mathrm{PH}$ dans un passé révolu, en rupture avec le repère origine de l'énonciation. Le repère temporel du procès "prend le pouvoir » dans la séquence (3) est $T 1=$ le 24 novembre 1965 tel que $\mathrm{T} 1$ est décroché du repère-origine $T o=9$ septembre 1997. De même, dans la séquence (4), on observe la même relation aoristique T1

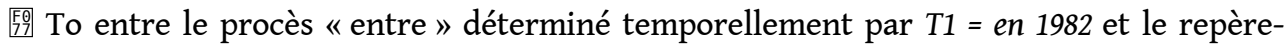
origine de l'actualité To = 19 septembre 1997 (date de parution de l'article). Les indications temporelles associées aux procès au $\mathrm{PH}$ ont pour fonction d'assigner une référence temporelle passée aux procès au $\mathrm{PH}$ et de marquer le décrochage temporel avec la situation d'énonciation origine définie par la date de parution du journal. Le présent, tiroir non autonome, ne tire sa valeur «historique» que du contexte qui construit le repérage aoristique des procès en décrochage avec le repère origine. Le décrochage entre le procès de l'événement et le procès de l'énonciation ne se fait pas de la même manière avec le PR de R, pour lequel on peut parler de repérage composite.

6 En plus du repérage aoristique, où le procès repéré fonctionne en rupture avec le repèreorigine (To), Culioli introduit l'existence d'un repère-origine fictif (noté $\mathrm{To}^{1}$ ) qui « fonctionne comme un repère-origine dédoublé, à la fois identifiable et non identifiable à 
Sito " (ibid: 186). La notion de repère-origine fictif s'accorde bien à la situation du reportage prototypique dans lequel le repère origine est translaté du repère de l'actualité conventionnel (To) indiqué par la date de parution du journal à un repère de l'actualité fictif (noté $\mathrm{To}^{1}$ ) correspondant au moment de l'événement. La relation entre le temps de l'événement $(\mathrm{Te})$ et le temps du repère origine $(\mathrm{To})$ est dite composite et notée étoile ( $\mathrm{Te}$ 詯己 $\mathrm{To}$ ), ce qui signifie que l'on a d'un côté $\mathrm{Te}=\mathrm{To}^{1}$ (les procès au PR de $\mathrm{R}$ coïncident avec le repère de l'actualité fictif) et de l'autre $\mathrm{Te}$ 膡 $\mathrm{T} 0$ (les procès au PR de $\mathrm{R}$ sont disjoints du repère de l'actualité conventionnel $\mathrm{To}$. Ce type de relation composite au repère-origine apparaît bien dans les séquences (5) et (6) :

(5) Premiers hommages à Kensington Palace, les derniers à l'abbaye de Westminter. Il est 9 heures samedi matin, et face à l'abbaye, le soleil commence à jeter son manteau de chaleur sur la place. Les « campeurs » encore engourdis par la fraîcheur de la nuit, tentent de se réchauffer les mains autour d'une tasse de café fumant.

L'Humanité 8/09/97, «Mère, divorcée, dépressive et amante: Diana était comme nous »

(6) En ce dimanche soir, la population de Mawpat converge vers l'église pour la messe, le village khasi est niché 1500 mètres d'altitude dans les montagnes du Meghalaya. Sur son piton, la petite chapelle en bois est noyée dans la brume. Meghalaya signifie "demeure des nuages ». Dans cette "Ecosse de l'Orient », les jeunes filles portent toutes d'élégants châles vichy. Avec leur type tibéto-birman, on les imaginerait mal vêtues du sari indien.

Quand l'office commence, l'Eglise est pleine à craquer. « Mawpat tout entier s'est converti » explique fièrement Eringson « Henri » Kharkongor, 67 ans, « moi, je suis catholique depuis que je me suis marié. J'ai suivi ma femme... ». Les Khasis forment une société matriarcale. La femme y est le chef incontesté de la famille. C'est par elle que se transmet la propriété.

Le Figaro 16/09/97, « Inde: conversions en pays khasi »

7 Il n'y a pas dans ces séquences de repérage absolu des procès comme avec le $\mathrm{PH}$ mais un repérage relatif par rapport à un repère-origine fictif donné comme contemporain d'une nouvelle origine énonciative. Ce repère-origine («samedi matin» dans 5 , «en ce dimanche soir » dans 6) est le point de départ de la succession événementielle des procès sans qu'il y ait mise en place d'une antériorité à To. Certes, le lecteur peut reconstruire cette antériorité en comparant la référence temporelle du repère-origine fictif à celle du repère-origine déterminé par la date de parution du journal, mais cette antériorité n'est pas donnée dans le texte comme avec le présent historique. Le repère de l'événement $(\mathrm{Te})$ est à la fois décroché du repère origine conventionnel To (tel que Te 医 To) mais conjoint au repère-origine fictif $\mathrm{To}^{1}\left(\right.$ tel que $\left.\mathrm{Te}=\mathrm{To}^{1}\right)$, d'où la relation composite $(\mathrm{Te}$ 餍 $\mathrm{To}$ ) entre le repère de l'événement et le repère-origine absolu.

Culioli (1978) introduit la notion de repère-origine fictif pour repérer les procès non validés des énoncés hypothétiques ou contrefactuels, mais il ajoute que le concept de repère-origine fictif vaut également pour des procès validés dans des récits d'événements attestés tels que les récits de reportage :

«On retrouve le concept de repère fictif dans des domaines variés: modalité naturellement, avec des prédicats tels que pouvoir, devoir, vouloir, avec les conditionnels etc., mais aussi, et de façon moins immédiate, dans des cas forts divers : nous avons parlé tout à l'heure des emplois ludiques, mais il existe aussi des emplois (si l'on peut se servir d'un terme aussi impropre) d'ordre fantasmatique, où le repère fictif fonctionne comme un repère origine dédoublé, à la fois identifiable et non identifiable à Sito. Dans les indications scéniques, le repère fictif va fonctionner comme un repère absolu, séparé du repère origine Sito. La situation est encore plus complexe dans la description d'une expérience par un expérimentateur s'adressant à un auditoire, au 
fur et à mesure du déroulement, ou dans le cas d'un reportage. »

Culioli $1978: 186$, nous soulignons

Culioli parle d'« emploi fantasmatique » du repère fictif, nous préférons parler de "valeur symbolique » car le nouveau repère de l'actualité indiqué par le moment de l'événement est une illusion qui fonctionne sur le mode du « comme si », tout comme le PR de R, dont le rôle est d'abolir la distance temporelle entre le réel et le symbolique de l'énonciation. Le PH fonctionne lui aussi sur le mode du « comme si », mais d'une autre manière : avec le $\mathrm{PH}$, les événements sont présentés comme s'ils étaient contemporains du moment de l'énonciation, avec le PR de $\mathrm{R}$ c'est l'énonciation qui est présentée comme si elle était contemporaine de l'événement. Mais alors que cette illusion référentielle est contredite par un repérage aoristique avec le $\mathrm{PH}$, elle est maintenue avec le PR de R par le truchement d'un repérage composite.

Pour formuler les choses autrement, on peut dire que ce qui est fictif dans le récit au PR de $\mathrm{R}$, ce n'est pas le présent mais le repère-origine de l'énonciation déplacé du repèreorigine conventionnel au repère-origine de l'événement. Le PR de $\mathrm{R}$ a donc bien une valeur actuelle et pourra être traduit dans une autre langue par un présent (Chuquet 1994). En revanche le récit au $\mathrm{PH}$ est généralement lui traduit par un tiroir passé, par exemple le preterit en anglais, ce qui montre sa valeur aoristique. En résumé, le PR de $\mathrm{R}$ est un vrai/faux présent, construit sur un repère-origine fictif, qui peut être transposé au passé, tandis que le PH construit sur un repérage aoristique constitue déjà une forme transposée d'un tiroir passé (passé simple, passé composé ou imparfait) et peut être considéré à ce titre comme un vrai/faux passé.

\section{La perspective temporelle du récit au présent de reportage}

11 On peut définir la perspective temporelle du récit (Genette 1972, Weinrich 1973) comme le rapport entre le temps du texte et le temps de l'événement. Le temps du texte peut coïncider avec le temps de l'événement, le précéder ou encore se situer longtemps après lui. La perspective temporelle sera dite simultanée si le temps du texte coïncide avec le temps de l'événement : cette perspective est exprimée par le présent. La perspective temporelle sera dite rétrospective si le temps du texte est postérieur au temps de l'événement: cette perspective est exprimée par les tiroirs du passé comme le passé composé, l'imparfait et le passé simple. Enfin la perspective temporelle sera dite prospective si le temps du texte anticipe sur le temps de l'événement : cette perspective est exprimée par le futur ou le conditionnel. Le propre du PH est d'abolir la perspective rétrospective du récit en faisant oublier le décalage temporel entre l'événement et son énonciation, mais cette perspective simultanée induite par l'emploi du présent est contredite par l'emploi du futur historique et d'indications temporelles autonomes qui ancrent le récit dans le révolu. Le PR de $\mathrm{R}$ en revanche ne pose pas l'antériorité des événements à To et entretient de ce fait une illusion de cotemporalité entre l'événement et son énonciation. Ces différences de perspectives exprimées par le PH et le PR de R apparaissent bien dans les séquences (7) et (8)

(7) D'un coup de pied, il ouvre la porte et pénètre dans le bureau du secrétaire du parti de la ville, un gros homme au teint lisse craint comme le sultan : « Afiatdine Djalilovitch , la lumière a été coupée dans le quartier arménien. Il y a une panique monstre. Prenons des mesures. " Nous sommes en 1988 dans l'un des quartiers 
arméniens de Bakou.

Le Figaro 9-10/05/98, "Le candidat du petit peuple »

(8) [...] Nous sommes dans la campagne, la nuit. Un hélicoptère militaire éclabousse

les bosquets de son phare blanc. Il est très bas, juste arraché à sa base. Tête

penchée, yeux plissés, Eamonn observe l'appareil un instant, évalue la distance en

silence, puis remonte le col de sa veste en disant: « dommage ».

Libération 25/09/97, « Le vertige des républicains irlandais »

Les deux constructions soulignées sont très similaires dans leur composition: elles comprennent un pronom déictique "nous ", un verbe d'état "sommes", un constituant spatial et un constituant temporel. L'unique différence est que le constituant temporel de (7) est une indication temporelle autonome ( en $1988 »)$ tandis que le constituant temporel de (8) est une indication temporelle relative («la nuit»). C'est cette unique indication temporelle qui modifie l'interprétation temporelle de la phrase : dans (7), la date « en 1988 » localise le procès antérieurement à To, par conséquent le présent de « nous sommes » a une valeur historique et la suite d'événements rapportés dans le reste de la séquence est interprétée a posteriori comme un récit rétrospectif. Dans (8), la seule information que donne l'indication temporelle «la nuit" est que le procès "nous sommes" lui est contemporain. Le procès n'est pas ancré dans le révolu et posé fictivement ( $c f$. section précédente) comme "actuel», ce qui implique une coïncidence entre le temps du récit et le temps de l'événement et donc une perspective temporelle simultanée. Le présent de (7) est un PH, celui de (8) un PR de R. Il n'y a pas que l'interprétation temporelle de la phrase soulignée qui soit modifiée par la nature de l'indication temporelle mais également le sens du déictique "nous». Dans (8), l'interprétation du pronom est ambiguë, «nous " pouvant représenter le narrateur en tant que personnage du récit plus un autre personnage du récit, ou le narrateur et le lecteur. Dans (7) en revanche, l'indication temporelle passée ( « en $1988 »)$ interdit d'envisager une co-présence entre le narrateur et le personnage désigné par «il», le déictique «nous » ne peut donc renvoyer qu'à la co-présence fictive entre le narrateur et le lecteur. Autrement dit, la construction "nous sommes en 1988 " n'a pas de signification référentielle et relève de ce que Vuillaume (1990) appelle la «fiction secondaire » lorsqu'à la scène de l'événement se superpose la scène de l'énonciation mettant en scène le narrateur et le lecteur. Cette construction contribue à dissocier les deux niveaux de l'histoire et du récit et à opposer la perspective simultanée de l'histoire à celle, rétrospective, du récit.

13 Le futur historique, souvent combiné au $\mathrm{PH}$, contribue également à dissocier les deux niveaux de l'histoire et du récit. La perspective temporelle du futur est normalement prospective puisque sa fonction est d'anticiper sur des événements non encore réalisés mais dans le cas du futur historique, il y a une double perspective prospective et rétrospective: le temps du texte anticipe sur le temps de l'événement, mais ces événements ont par ailleurs déjà été réalisés dans le passé. On peut donc parler d'un conflit de perspectives temporelles dans le récit au présent historique dans lequel plusieurs perspectives coexistent : une perspective simultanée avec l'emploi du PH, une perspective rétrospective avec l'emploi d'indications temporelles autonomes marquant le décrochage des procès par rapport à To, et enfin une perspective prospective/ rétrospective avec l'emploi du futur historique. Pour que le PH remplisse effectivement les valeurs expressives qu'on lui attribue traditionnellement («saisie sur le vif », effet de " témoignage en direct»), il faudrait qu'il fonctionne avec une combinatoire temporelle différente qui ne contredise par la perspective temporelle simultanée associée à l'emploi 
du présent. Cette condition semble remplie avec l'utilisation du PR de $\mathrm{R}$ qui permet véritablement un « récit en direct » d'événements.

Le PR de $R$ présente en effet les procès dans une perspective simultanée qui n'est contredite ni par les indications temporelles du contexte ni par les alternances temporelles avec lesquelles ce tiroir entre en corrélation. A l'inverse des séquences au $\mathrm{PH}$, les séquences au PR de R ne comportent pas d'indications temporelles autonomes mais des indications déictiques qui, pour être déterminées temporellement, ont besoin d'être rapportées à un autre point de référence, en l'occurrence le repère-origine fictif.

(9) Accoudé au comptoir d'un pub, le vieil Olivier raconte son fils tué Franck, tué il y a vingt-deux ans par une bombe républicaine. Son coupé, déformé par la réception approximative, le téléviseur renvoie l'image tremblée de Gerry Adams. "C'est lui qui m'a enlevé mon petit ", dit-il simplement.

Ce soir, l'inquiétude est fleur de la rue. Ce n'est pas un éventuel retour de la violence qui inquiète, mais de quoi demain sera fait. Ici, à l'angle d'un trottoir peint en drapeau britannique, là aux caisses de petit supermarché, devant les grilles noircies de bureau de chômage, attablés pour le bingo sans joie du mardi soir à Tiger Bay, des petits bouts de femme, des gueules cassés d'hommes s'interrogent. Ils sont protestants, se jurent mal aimés, piégés par le jeu des politiciens, abandonnés. Libération 24/09/97, «Ceux qui ont souffert savent qu'il est temps de penser » (10) Sarno, samedi à midi. Le soleil tape dur, il fait une chaleur torride. En bas, la ville, en haut la montagne où se dessinent treize grands serpents. Treize traînées d'où a littéralement déboulé un grand fleuve de boue il y a maintenant cinq jours. Une avalanche de terre mélangée à l'eau des grandes pluies qui s'est déversée sur cette petite ville nichée au pied de la montagne à quelques kilomètre de Pompéi et du Vésuve.

Samedi 9 mai, il est midi. La route qui traverse Sarno est pleine de boue : la circulation est dense. Les camions de l'armée, les tracteurs réquisitionnés pour déblayer la ville entrent et sortent dans un "via vai" continu. Les vigiles chargés de la circulation portent tous les masques ou plus simplement un foulard noué autour du visage en guise de protection. [...] Nous grimpons le long de la route, vers le cœur de la ville, le cœur du fleuve boueux. Un oranger se dresse en signe d'espoir devant une maison blanche ayant échappée au désastre. Nous reprenons la route vers Quindici [...].

L'Humanité 11/05/98 , « L'odeur terrible qui s'élève du ventre de la boue de Sarno »

Les expressions déictiques localisent les procès par rapport au repère-origine de l'énonciation mais le repère-origine des textes de reportage étant fictif (cf. section 2), il est difficile de rapporter les déictiques à la temporalité extralinguistique et d'assigner une référence temporelle aux procès. Ainsi «ce soir» dans (9) renvoie au soir de la rencontre entre le reporter et « le vieil olivier » sans que l'on puisse dater précisément la rencontre. Seule l'indication déictique de (10) «samedi à midi" reçoit une référence temporelle tardive avec la reprise anaphorique du syntagme complété d'une date "samedi 9 mai, il est midi ». Cette dernière indication temporelle est toutefois semiautonome (la date ne mentionne que le jour et le mois sans l'année) et seule notre connaissance extralinguistique et la référence à la date de parution du journal nous permet d'inférer qu'il s'agit du 9 mai 1998. On peut noter que le syntagme qui introduit la séquence (10) («Sarno, samedi à midi ») avec mention du lieu et de la date (même si la date est incomplète) joue le rôle de la formule de « suscription à l'ancienne » tel que nous l'avons rencontrée dans les quotidiens des années 50 et 60 . Il est toutefois rare que le repère-origine fictif soit aussi explicite : il s'agit en effet de faire oublier que le reportage est un texte différé et l'absence d'indications temporelles autonomes permet de maintenir l'illusion d'une perspective simultanée du récit, déjà induite par l'emploi du 
présent. Ce qui est signifiant avec le PR de R n'est pas la temporalité officielle telle qu'elle a pu être reconstituée après coup (et signifiée par les indications temporelles autonomes) mais la temporalité plus subjective de qui vit l'événement en direct.

Tout comme les indications temporelles non autonomes ne contredisent par la perspective simultanée du récit au $\mathrm{PR}$ de $\mathrm{R}$, les alternances temporelles corrélées à ce tiroir, en ne dissociant pas la temporalité du récit et la temporalité de l'événement, maintiennent «l'effet de direct». En fait, le PR de R est un tiroir très récurrent; les alternances temporelles sont rares et, quand elles se produisent, ce n'est pas avec le futur historique mais avec le PC :

(11) [...] Le président repart. La porte de la prison s'ouvre. Les prisonniers sortent un à un. Cheikh apparaît: le bras droit en écharpe et le crâne emmailloté dans un linge blanc. Sa mâchoire a été fracassée et les coups ont fait perdre à ses yeux toute symétrie. La terreur achève de les rendre fous.

Le Figaro 23/09/97, « Anjouan, la victoire en déchantant »

(12) Le ferry vient de s'immobiliser dans un nuage d'écume, au milieu du détroit de Malaca. Remue-ménage dans la salle de pilotage. Un gros point vert est apparu depuis quelques minutes sur la droite de l'écran radar et se rapproche de la position du bateau. Mâchoire serré, le capitaine assisté de cinq marins, scrute la mer [...] Les dizaines de voyageurs qui s'entassent au milieu des enfants et paquets ont également tourné en silence leur regard anxieux vers les flots gris [...] Le petit bateau tangue un moment sur le sillage du montre noir qui a déjà disparu dans le brouillard. Le capitaine essuie ses lunettes.

Libération 29/09/97, « Dumaï, un mois sans soleil »

(13) Drôle de route. Drôle d'ambiance. Il est midi, et cette campagne des pommiers en fleurs est trop silencieuse. Qu'y a-t-il derrière les hauts murs de ces fermes albanaises aux volets clos? On avance doucement, le ventre noué, sachant que les plaques belgradoises de notre voiture de location ne nous désignent pas comme des «amis » potentiels. On est entré en territoire contrôlé par l'UCK, la mystérieuse Armée de libération du Kosovo.

$\mathrm{Au}$ loin, le ronronnement d'un moteur de tracteur, signe rassurant que la vie, ici, ne s'est pas totalement arrêtée. Un petit village apparaît sur la droite, à cent mètres à l'écart de notre route caillouteuse, traversant d'est en ouest ce triangle rebelle de $400 \mathrm{~km}^{2}$ (avec pour sommets les villes de Pec, de Djacovica et de Klina), où les policiers serbes ne s'aventurent plus depuis un mois. Si l'on en croit la carte d'étatmajor, la ville s'appelle Lumbrali.

La place centrale fourmille de paysans en armes, en pleine discussion, les yeux rivés sur le balcon en béton d'une maison en construction. Une mitrailleuse lourde y a été disposée, et chacun semble avoir son idée sur le meilleur angle de tir en direction de la route.

Le Figaro 5/05/98, « Kosovo : la rébellion inextinguible»

17 Le PC n'est pas utilisé dans ces séquences comme temps narratif du passé mais dans sa valeur d'accompli ou d'antériorité à To. Les procès de (12) «est apparu depuis quelques minutes » « ont également tourné » « a déjà disparu » marquent l'antériorité immédiate par rapport aux procès au présent. La périphrase verbale «vient de s'immobiliser » dans (12) a également une valeur de passé récent. Dans (11) et (13), les procès au PC «a été fracassé », « ont fait perdre » « est entré » « a été disposé » combinent les deux valeurs du PC de discours, la valeur d'accompli et d'antériorité par rapport au présent de la narration. Tous les procès au $\mathrm{PC}$ sont donc liés temporellement au repère de l'actualité et sont dans le même référentiel temporel que les procès au PR. Dans les séquences au PR de $\mathrm{R}$, l'illusion de co-temporalité avec l'événement est maintenue jusqu'au bout : aucun élément, indication temporelle autonome ou procès au futur historique ne vient interrompre le fil de l'histoire pour en rappeler le caractère rétrospectif. L'énonciateur ne 
se pose pas en narrateur omniscient comme pour le futur historique mais adopte le point de vue du lecteur, c'est-à-dire que la temporalité du récit n'anticipe pas sur celle de l'événement. L'«illusion référentielle » entretenue par le PR de R est que les événements se déroulent au fur et à mesure où on les raconte, par conséquent on ne peut avoir la prescience des événements à venir (absence de futurs historiques) même si on connaît ceux qui ont déjà été actualisés par rapport au repère de l'actualité du reportage, d'où les occurrences de PC.

Le PR de $\mathrm{R}$ abolit la dualité temporelle du récit pour mettre en avant la temporalité existentielle de l'événement. Le PR de R, plus encore que le PH assure une fonction expressive car il inscrit les procès dans un temps qui n'a rien à voir avec le temps chronologique du calendrier, c'est le temps du «vécu-perçu» qui donne à voir les événements. C'est ce point de vue perceptuel sur les procès que je vais maintenant développer.

\section{La relation point de vue}

Le concept de relation point de vue (Rpv) a été introduit par Vogeleer (1992), pour décrire le type d'accès cognitif qui relie un individu appelé individu point de vue (l'Ipv) à l'information décrite dans une phrase. Cette information peut être obtenue par observation et dans ce cas la relation point de vue est dite perceptuelle, ou l'information peut être obtenue en vertu d'un savoir préalable et la Rpv sera dite épistémique. A la différence des études sur l'évidentialité (Dendale 1994) ou le médiatif (Guentcheva 1994), le concept de relation point de vue vise moins à mettre en évidence la source de l'information (qui peut être obtenue par observation, inférence ou être empruntée à un tiers) qu'à décrire la nature (perceptuelle ou épistémique) de l'accès cognitif à l'information.

La relation point de vue se présente donc sous la forme d'une relation cognitive de type épistémique fondée sur la relation SAVOIR, ou sous la forme d'une relation cognitive de type perceptuel fondée sur la relation VOIR. Les deux Rpv se caractérisent par les paramètres spatio-temporels Tpv et Lpv (temps de point de vue et lieu de point de vue) qui ne sont pas nécessairement identiques au temps To et au lieu Lo du repère-origine. Les temps verbaux reflètent ces différentes relations point de vue épistémique ou perceptuelle. Ainsi, avec le $\mathrm{PH}$, l'accès à l'information est de nature épistémique fondé sur la relation savoir : autrement dit le journaliste utilise le PH pour les événements dont il n'a pas été le témoin mais qu'il rapporte en vertu d'un savoir préalable. Ces événements sont rapportés à partir du repère de l'actualité conventionnel (la date de parution du journal) et le temps de point de vue correspond à ce repère $(\mathrm{Tpv}=\mathrm{To})$. Avec le $\mathrm{PR}$ de $\mathrm{R}$, le journaliste rapporte ce qu'il a vu ou entendu en vertu d'un Rpv perceptuelle. Ce type de Rpv est possible dans le reportage parce que le journaliste a été le témoin des événements qu'il rapporte comme l'indique la formule de suscription (De notre correspondant à X). Le temps et le lieu de point de vue se confondent avec le temps et le lieu de l'événement (Tpv $=\mathrm{Te}$ ). Ces différences de Rpv entre PH et PR de R sont bien reflétées dans les séquences (14) et (3):

(14) Les orateurs se succèdent. Les «pro» libération et les «anti». La foule applaudit les uns, insulte les autres. La scène est à mi-chemin entre l'émeute et la kermesse populaire. Des jeunes entonnent des slogans font chanter les femmes. Le Figaro 23/09/97, «Anjouan : la victoire en déchantant » 
(3) C'est le 24 novembre 1965 que Joseph-Désiré Mobutu, alors âgé de trente-cinq ans, prend le pouvoir en renversant le président Joseph Kasavubu. L'ancien sergent de la «Force publique » du Congo sous colonisation belge, devenu journaliste, secrétaire particulier de Patrice Lumumba puis chef d'état-major des armées, tisse peu à peu sa toile sur l'ensemble du pays. Celui qui prétend incarner la nation élimine la vieille garde politique, interdit les partis, suspend le droit de grève, et s'applique à instituer l'unité du pays à son profit personnel.

L'Humanité 9/09/97, «La mort du maréchal-gangster Mobutu »

21 La séquence (14) est la description d'une suite d'événements du point de vue d'un observateur « naîf » (l'Ipv) qui décrit ce qu'il voit sans connaître à un moment donné de l'observation, la suite des événements, ni les relations causales entre ceux-ci. La situation $e<$ les orateurs se succèdent $>$ est décrite du point de vue d'un individu qui voit la situation se dérouler sous ses yeux, la relation point de vue est donc perceptuelle. La Rpv <voir> n'est possible que si l'Ipv est lié spatialement à $e$. L'Ipv est ici absent de l'énoncé (absence de pronom déictique) mais on sait par la formule de suscription qu'il s'agit du journaliste présent sur les lieux de la situation qu'il décrit, d'où Lpv = Le, le lieu du point de vue est aussi le lieu de l'événement. L'Ipv accède perceptuellement à $e$ alors que $e$ est en cours, le temps du point de vue correspond donc plus ou moins au temps de l'événement (Tpv = Te). Les propriétés physiologiques de la vision humaine font que la situation $e$ perçue par l'Ipv doit être limitée à l'intérieur d'un intervalle relativement court localisé à l'intérieur d'une journée. C'est le cas de la situation $e$ <les orateurs se succèdent> qui, bien que désignant une suite d'actions itératives, est limitée dans le temps. Sur le plan temporel, l'information communiquée par la situation $e$ est différée et donc Te $\neq$ To, néanmoins nous avons vu que dans le reportage au présent ( $c f$. section 2), le repère-origine (To) est déplacé à un repère-origine fictif $\left(\mathrm{To}^{1}\right)$ tel que $\mathrm{To}^{1}=\mathrm{Te}$. On a donc la double équivalence $\mathrm{Tpv}=\mathrm{Te}$ et $\mathrm{Te}=\mathrm{To}^{1}$. Le temps du point de vue est identique au temps de l'événement qui est lui même identique au temps du repère-origine fictif. Le lieu du repère-origine fictif $\left(\mathrm{Lo}^{1}\right)$ étant aussi celui de l'événement (Le), on a l'équivalence $: \mathrm{Lpv}=\mathrm{Le}=\mathrm{Lo}^{1}$.

Dans (3), la Rpv est différente. L'Ipv dit que $p<$ Mobutu prend le pouvoir> non pas parce qu'il voit Mobutu prendre le pouvoir mais parce qu'il le sait. C'est le contenu propositionnel de la phrase qui indique que la Rpv est épistémique : on pourrait imaginer un énoncé avec le même procès <prendre le pouvoir> basé sur la Rpv perceptuelle voit (Ipv, e) du type «il prend le pouvoir » (impliquant que l'Ipv voit « il » prendre le pouvoir) mais l'ajout d'une indication temporelle autonome "c'est le 24 novembre 1965 », des indications patronymiques" "Joseph-Désiré Mobutu» "Joseph Kasavubu» et de la construction détachée "alors âgé de 35 ans " fait basculer le même énoncé dans le domaine épistémique (on ne voit pas une date, on ne voit pas X avoir 35 ans). Dans le cadre de cette Rpv épistémique, l'Ipv n'a pas un accès direct à la situation $e<$ Mobutu prend le pouvoir $>$ mais à une représentation conceptuelle de $e$ que l'on note $p$. Autrement dit, avec la Rpv épistémique, l'Ipv n'accède pas directement à une situation comme avec la Rpv perceptuelle mais à une proposition $p$ qui représente le contenu de l'information.

Nous avons noté dans la section 2 que le $\mathrm{PH}$ était corrélé à des indications temporelles autonomes permettant de localiser les procès. Le contenu de ces indications temporelles autonomes est fondé sur la Rpv épistémique. En effet, à la différence d'un lieu ou d'un individu, un point ou un intervalle temporel est inaccessible à la perception :

«On ne peut VOIR un point, un intervalle temporel $t$ porter une certaine date, ni simplement VOIR $t$, ni VOIR t être associé à un événement e. Il en résulte que l'accès à l'information sur le temps contenue dans le constituant temporel, ne peut être qu'épistémique, puisque de par sa nature, cette information n'est accessible qu'en 
vertu de la relation savoir ".

(Vogeleer $1994: 75$, souligné par l'auteur.) indication temporelle relative ${ }^{4}$ localise le temps Te du procès « a commencé » par rapport au temps To du repère de l'actualité (la date de parution du journal). Cette référence explicite au repère de l'actualité signale, d'une part, que le temps du point de vue (Tpv) est identique au temps To du repère de l'actualité et, d'autre part, que le temps du point de vue n'est pas identique au temps Te de l'événement, ce que confirme également la désinence passée du procès " a commencé ». La séquence se poursuit avec des procès au PH accompagnés des indications temporelles autonomes « en 1989 » et « en 1992-1993 et jusqu'en $1995 \%$. Ces indications temporelles autonomes signalent le décrochage aoristique des situations et, malgré la désinence PR des procès, elles indiquent que le temps de l'événement n'est pas identique au temps du repère de l'actualité ( $\mathrm{Te} \neq$ To). L'accès au temps Te de l'événement ne peut être attribué qu'à un Ipv qui se trouve en dehors du monde du texte (le journaliste) et qui introduit le temps à partir de To parce qu'il SAIT que le temps de l'événement est « en 1989 » $(\mathrm{Tpv}=\mathrm{To}, \mathrm{Tpv} \neq \mathrm{Te})$. Par ailleurs, l'intervalle de temps défini par les deux indications temporelles « en 1992-1993 jusqu'en 1995 » a une durée de trois ans, cette durée est inaccessible à la perception et ne peut se fonder que sur une relation épistémique. La séquence se conclut avec des procès au futur historique (« demandera » « recevra») qui dissocient temps de l'événement et temps du récit ( $\mathrm{Te} \neq \mathrm{Tpv}$ ) et auxquels l'Ipv n'a accès que parce ce qu'il SAIT que ces procès ont déjà été actualisés dans le passé ( $\mathrm{Te} \neq \mathrm{To}$ ). Les constituants temporels utilisés avec le $\mathrm{PH}$, qu'il s'agisse d'indications temporelles ou de procès au futur historique, signalent que l'émetteur a accès à l'information $p$ ( $p$ étant une représentation conceptuelle de $e$ ) à partir du repère de l'actualité et en vertu de la relation épistémique SAVOIR.

A la différence du PH, le PR de $\mathrm{R}$ se combine avec des indications temporelles non autonomes fondées sur une Rpv perceptuelle :

(16) Premiers hommages à Kensington Palace, les derniers à l'abbaye de Westminter. Il est 9 heures samedi matin, et face à l'abbaye, le soleil commence à jeter son manteau de chaleur sur la place, Les «campeurs» encore engourdis par la fraîcheur de la nuit, tentent de se réchauffer les mains autour d' une tasse de café fumant. 
L'Humanité 8/09/97, «Mère, divorcée, dépressive et amante: Diana était comme nous »

(17) Drôle de route. Drôle d'ambiance. Il est midi, et cette campagne des pommiers en fleurs est trop silencieuse. Qu'y a-t-il derrière les hauts murs de ces fermes albanaises aux volets clos? On avance doucement, le ventre noué, sachant que les plaques belgradoises de notre voiture de location ne nous désignent pas comme des «amis » potentiels. On est entré en territoire contrôlé par l'UCK, la mystérieuse Armée de libération du Kosovo [...].

Le Figaro 5/05/98, « Kosovo : la rébellion inextinguible » d'autres éléments du co-texte tels que les segments descriptifs, mais il faudrait citer également l'emploi des présentatifs, les verbes de vision et de monstration, le recours privilégié au discours direct, les phrases existentielles de type "apparait un X» (Vogeleer 1992), sans oublier l'hyperstructure (Grosse et Seiblod 1996) du reportage (photos, cartes géographiques, etc.), tous ces éléments participant au «montage audiovisuel » de l'information (Facques 2000 et 2001) dans ce genre de textes.

\section{Conclusion}

Je pense avoir montré que le PR de $\mathrm{R}$ n'était pas réservé aux récits oraux et que son emploi dans la presse écrite pouvait être distingué du PH sur le plan du repérage des procès, de la perspective temporelle du récit et de l'accès cognitif à l'information. On peut dès lors définir le présent de reportage comme un présent construit sur un repèreorigine-fictif, donnant une perspective simultanée sur l'événement et privilégiant un 
accès perceptuel à l'information. Il reste que le $\mathrm{PH}$ et le PR de R sont des catégories interprétatives qui ne peuvent apparaitre qu'en discours avec l'étude des indications temporelles non verbales, des alternances avec lesquelles les procès au présent entrent en corrélation et des caractéristiques sémantiques des procès. Cela explique que la séparation ne soit pas toujours facile à établir entre ces deux emplois de présent : le $\mathrm{PH}$ peut tendre vers le PR de R, pour peu que sa combinatoire temporelle soit différente, et le PR de R peut se rapprocher du PH lorsqu'il est associé à des futurs historiques ou à des procès fondés sur une relation point de vue épistémique.

31 Le PR de R se rencontre de façon privilégiée dans les reportages de presse écrite car il permet de compenser les caractéristiques situationnelles de ce genre où le journaliste se pose en témoin de l'événement (formule de suscription) mais ne peut le retransmettre directement du fait du caractère différé de l'information. Le «jeu » de PR de R est alors d'inscrire l'événement dans une équivalence spatiale et temporelle avec la situation du lecteur et de masquer ainsi le caractère différé de l'information et son éloignement géographique. Par ailleurs, le $\mathrm{PR}$ de $\mathrm{R}$ qui est fondé sur un accès perceptuel à l'information convient au reportage, genre descriptif qui privilégie le FAIRE-VOIR sur le FAIRE-SAVOIR. Le fait de langue est ainsi étroitement lié au fait générique même si le PR de R peut aussi se rencontrer dans d'autres genres de la presse écrite (portrait, interview, chronique, etc.) où il s'agit de créer un «effet reportage» ou de faire croire à une actualité de l'événement.

\section{BIBLIOGRAPHIE}

Chuquet, H. (1994). Linguistique contrastive et traduction. Leprésent de narration en anglais et en français, Ophrys

Culioli, A. (1978). « Valeurs aspectuelles et opérations énonciatives :l'aoristique », in J. David et R. Martin(éds), Actes du colloque sur la notion d'aspect, Publication de l'Université de Metz :181-194

Dendale, P. et Tasmowski, L. (1994). « L'évidentialité ou lemarquage des sources du savoir », Langue Française, $102: 37$

Facques, B. (2000). «Le reportage de presse écrite : un genre audiovisuel? », in Changing landscapes in language and language pedagogy : Text,orality and voice, M.N Guillot and M.M Kenning (eds), London: CILT/AFLS, 123-149

Facques, B. (2001). La Variation temporelle entre langue et discours : une étude des récurrences, alternances et ruptures temporelles dans les textes de reportage de la presse quotidienne française, thèse de doctorat en co-tutelle sous la direction de Sophie Moirand et Carol Sanders, Paris III et Université du Surrey

Genette, G. (1972). Figures III, Editions du Seuil

Guentcheva, Z. (1994). « Manifestations de la catégorie du médiatif dans les temps français », Langue Française 102 : 8-23 
Mellet, S. (1998). « Présent et présentification : un problème d'aspect », in Temps et Discours, Sv.Vogeleer ; A. Borillo ; C. Vetters ; M. Vuillaume (éds), Louvain-la Neuve : Peeters, 203-213

Mellet, S. (2000). « Le présent », Travaux de Linguistique 40 : 97-111

Riegel, M. et alii (1994). Grammaire méthodique du français, PUF

Tasmowski-De Ryck, L. (1985). «L'imparfait avec et sans rupture », Langue Française 67 : 59-77.

Vogeleer, S. (1992). «La relation point de vue et son application aux phrases existentielles initiales ", in Enonciation et parti pris, W. de Mulder ; F. Schuerewegen ; L. Tasmowski-De Ryck (éds), Amsterdam : Rodopi, 349-355.

Vogeleer, S. (1994). «Le point de vue et les valeurs des temps verbaux », Travaux de Linguistique $29: 39-58$

Voirol, M. (1995). Guide de la rédaction, Paris : Centre de formation et de perfectionnement des journalistes (CFPJ)

Vuillaume, M. (1990). Grammaire temporelle des récits, Editions de Minuit

Weinrich, H. (1973). Le Temps, (trad. fr., éd. orig. 1964), Seuil

\section{NOTES}

1. Libération ne figure pas ici car ce journal n'a été créé qu'en 1973.

2. L'indication patronymique complète implique une distance spatiale et temporelle entre l'Ipv et le personnage décrit. Lorsqu'une personne est décrite d'un point de vue perceptuel, elle n'est pas nommée ou par son prénom seulement.

3. Citons cette remarque de Wagner et Pinchon (1962: 377) qui lie implicitement futur historique et point de vue épistémique sur le procès «Par ce tour, le narrateur crée un décalage expressif dans un récit dont les verbes sont à un temps du passé ou du présent historique. Fort de ses connaissances, il évoque au moyen du futur des faits qui sont passés par rapport à lui, mais qui étaient encore à venir au moment où se situe l'histoire racontée. »

4. Rappelons que les indications temporelles relatives ou non autonomes s'opposent aux indications temporelles autonomes en ce qu'elles nécessitent pour recevoir une référence temporelle d'être rapportées à un autre constituant temporel présent dans le cotexte, ou au repère temporel de la situation d'énonciation.

5. Il s'agit d'une séquence d'incipit, ces démonstratifs ne peuvent par conséquent avoir une valeur anaphorique et renvoient nécessairement à la situation de l'Ipv.

\section{RÉSUMÉS}

Cet article présente une nouvelle description du présent narratif et propose de distinguer dans les récits d'événements attestés entre le "présent de reportage » et le "présent historique ». L'étude de la combinatoire temporelle (alternances temporelles et indications temporelles non verbales) du présent en contexte narratif permet de contraster le présent de reportage et le présent historique sur le plan du repérage des procès, de la perspective temporelle du récit et de 
l'accès cognitif à l'information. Alors que le présent historique se caractérise par un repérage aoristique, on peut parler de repérage composite (Culioli 1978) pour le présent de reportage. Ces deux valeurs de présent se distinguent également sur le plan de la perspective temporelle du récit, simultanée pour le présent de reportage, simultanée et rétrospective en ce qui concerne le présent historique. Enfin présent historique et présent de reportage n'offrent par le même type d'accès cognitif à l'information : alors que le premier suppose un point de vue épistémique sur l'information, le second est lui fondé sur un accès perceptuel à l'information.

This article puts forward a new description of the narrative present and distinguishes in journalistic discourse between "reporting present" and historical present. These two forms differ in terms of their temporal perspective, in the way they relate to events and in the way they give access to information from a cognitive point of view. The temporal perspective is simultaneous only with the "reporting present", simultaneous and retrospective with the historical present. The relationship between the event and the original point is aoristic with the historical present, composite with the "reporting present". Finally historical present involves an epistemic access to information whereas "reporting present" is based upon a perceptual point of view.

\section{INDEX}

Mots-clés : présent de reportage, présent historique, repérage temporel, perspective temporelle, accès cognitif à l'information.

\section{AUTEUR \\ BÉNÉDICTE FACQUES}

Université du Surrey 